\title{
Unterschiede in der Wahrnehmung positiven und negativen Feedbacks von Mädchen und Jungen im Leseunterricht der Grundschule
}

\author{
Fabian Hoya (D)
}

Eingegangen: 13. Februar 2020 / Überarbeitet: 10. März 2021 / Angenommen: 18. März 2021 / Online publiziert: 7. April 2021

(C) Der/die Autor(en) 2021

Zusammenfassung In zahlreichen Untersuchungen konnten geschlechtsspezifische Unterschiede im Lesen bei Kindern im Grundschulalter belegt werden. Im Detail weisen Mädchen im Vergleich zu Jungen sowohl bessere Leistungen als auch eine höhere Motivation und stärker ausgeprägte Selbstkonzepte auf. Diese leistungsbezogenen Unterschiede wie auch differente Erwartungen, die Lehrerinnen und Lehrer an Mädchen und Jungen im Leseunterricht stellen, können zu einem unterschiedlichen Feedbackverhalten führen, das sie gegenüber Schülerinnen und Schülern zeigen. In der vorliegenden Untersuchung wird der Frage nachgegangen, ob Mädchen und Jungen sich vor diesem Hintergrund in der Wahrnehmung des erteilten positiven und negativen Feedbacks ihrer Lehrkräfte im Leseunterricht unterscheiden. Zusätzlich wird untersucht, ob und inwiefern Unterschiede in dem von Grundschulkindern perzipierten Feedback ihrer Lehrkräfte über ihr Geschlecht, ihre Leistungen, ihre Motivation, sowie ihre Selbstkonzepte erklärt werden können. Dabei wurden $N=665$ Schülerinnen und Schüler der dritten und vierten Jahrgangsstufe zu dem von ihnen perzipierten positiven und negativen Feedback ihrer Lehrkräfte im Leseunterricht der Grundschule befragt. Darüber hinaus wurden Instrumente zum lesebezogenen Selbstkonzept, zur intrinsischen Lesemotivation und zum Leseverständnis der Kinder eingesetzt. Die Untersuchungsbefunde belegen, dass Mädchen im Vergleich zu Jungen signifikant häufiger positives Feedback und signifikant seltener negatives Feedback ihrer Lehrkräfte im Leseunterricht der Grundschule wahrnehmen. Insgesamt zeigt sich, dass sich geschlechtsspezifische Unterschiede im wahrgenommenen positiven und negativen Feedback von Grundschulkindern stärker über leistungsbezogene Variablen als über ihr Geschlecht erklären lassen.

F. Hoya $(\bowtie)$

Institut für Erziehungswissenschaft, Universität Paderborn, Warburger Straße 100, 33098 Paderborn, Deutschland

E-Mail: fabian.hoya@campus.upb.de 
Schlüsselwörter Geschlechterunterschiede $\cdot$ Feedback · Leseleistung · Motivation · Selbstkonzept · Grundschule

\title{
Differences in girls' and boys' perception of positive and negative feedback in primary school reading lessons
}

\begin{abstract}
Numerous studies indicate gender differences of primary school children in reading. In comparison to boys, girls show a higher performance, a higher motivation as well as higher self-concepts in reading. These performance-related differences, as well as the different expectations which primary school teachers place on girls and boys in reading lessons, can lead to an unequal teacher feedback behavior towards female and male students. In the present study, it is investigated whether girls and boys differ in their perception of teachers' positive and negative feedback in reading lessons. In addition, it is examined if differences in the feedback perceived by primary school children can be explained by their gender, their performance, their motivation as well as their self-concepts in reading. Therefore, $N=665$ third and fourth graders were asked about their perceived positive and negative feedback of their teachers in primary school reading lessons. Moreover, instruments for children's reading-related self-concept, their intrinsic reading motivation and their reading comprehension were used. The results of the study show that girls (in comparison to boys) are significantly more likely to perceive positive feedback and significantly less likely to perceive negative feedback from their teachers in reading lessons. Overall, it can be highlighted that gender-specific differences in primary school children's perceived positive and negative teacher feedback can be explained more by performance-related variables than by their gender.
\end{abstract}

Keywords Gender differences · Feedback · Reading performance $\cdot$ Motivation · Self-concept $\cdot$ Primary school

\section{Problemstellung}

Die Befunde der Internationalen Grundschul-Lese-Untersuchungen (IGLU) der vergangenen Jahre zeigen ein problematisches Bild an Grundschulen auf. Obwohl der Lesekompetenz eine Schlüsselrolle für schulische und außerschulische Lernprozesse zukommt und diese eine zentrale Komponente für die gesellschaftliche Partizipation darstellt (vgl. McElvany 2008), konnten in vielen Untersuchungen deutliche Unterschiede in den Lesekompetenzen von Mädchen und Jungen im Grundschulalter belegt werden (vgl. z. B. Bos et al. 2012; Hornberg et al. 2007; McElvany et al. 2017). Einen möglichen Erklärungsansatz für die bestehenden Unterschiede von Mädchen und Jungen im Lesen bilden gesellschaftlich vorherrschende Stereotype, die Kindern in Abhängigkeit ihres Geschlechts von der Gesellschaft zugeschrieben werden (vgl. Faulstich-Wieland 1995; Hannover et al. 2014). Mit Blick auf den Grundschulunterricht ergibt sich hieraus die Problematik, dass Lehrkräfte unterschiedliche Erwartungen an Mädchen und Jungen im Lesen stellen. Diese Erwartungen von Lehrkräften, im Sinne von Prognosen zur Leistungsentwicklung ihrer Schülerinnen 
und Schüler, können dabei ihre Interaktion mit den Lernenden im Unterricht beeinflussen. Hieraus können ungleiche Voraussetzungen für den Lesekompetenzerwerb von Mädchen und Jungen in der Grundschule resultieren.

\section{Geschlechtsspezifische Unterschiede von Kindern im Grundschulalter}

\subsection{Bestehende domänenspezifische Unterschiede von Mädchen und Jungen im Grundschulalter}

In einer Vielzahl an empirischen Untersuchungen wurden in den vergangenen Jahren die domänenspezifischen Kompetenzen von Mädchen und Jungen im Grundschulalter für den sprachlichen sowie mathematisch-naturwissenschaftlichen Bereich untersucht. Diese belegen (mit wenigen Ausnahmen: vgl. z. B. Gentrup et al. 2018; Helwig et al. 2001), dass sich Mädchen und Jungen im Grundschulalter in ihren sprachlichen sowie mathematisch-naturwissenschaftlichen Kompetenzen und selbstbezogenen Kognitionen unterscheiden. Im Detail zeigen die Befunde aus den Internationalen Grundschul-Lese-Untersuchungen (IGLU) sowie einer Vielzahl weiterer Studien einheitlich signifikant höhere Leseleistungen von Mädchen im Vergleich zu Jungen (vgl. z. B. Bos et al. 2012; Ehm et al. 2011; Hellmich 2008; Hornberg et al. 2007; Kessels und Heyder 2017; McElvany et al. 2017; Niklas und Schneider 2012).

Darüber hinaus kann anhand von zahlreichen weiteren Befunden aufgezeigt werden, dass die lesebezogenen Kognitionen von Kindern im Grundschulalter zusätzlich auf signifikantem Niveau zugunsten der Mädchen differieren. Diese weisen demnach eine stärker ausgeprägte Lesemotivation sowie höhere lesebezogene Selbstkonzepte auf (vgl. z. B. Ehm et al. 2011; Hornberg et al. 2007; McElvany et al. 2017; McGeown 2015; McGeown et al. 2011; Niklas und Schneider 2012). Dagegen verfügen Jungen über bessere Leistungen, eine höhere Motivation als auch stärker ausgeprägte Selbstkonzepte im mathematisch-naturwissenschaftlichen Bereich (vgl. z. B. Ehm et al. 2011; Gentrup et al. 2018; Lindberg et al. 2013; Niklas und Schneider 2012; Wendt et al. 2016).

Die zuvor berichteten geschlechtsspezifischen Unterschiede von Grundschulkindern weisen dabei unter Bezugnahme auf Cohen $(1988,1992)$ kleine bis mittlere Effektstärken hinsichtlich der Leistungen im Lesen (Ehm et al. 2011: $\eta^{2}=0,01$; Hellmich 2008: $d=0,23$; Niklas und Schneider 2012: $d=0,24-0,28)$ sowie im Fach Mathematik (Ehm et al. 2011: $\eta^{2}=0,01$; Niklas und Schneider 2012: $d=0,21-0,59$ ) auf. Ebenso zeigen sich Effektstärken auf kleinem bis mittlerem Niveau für die lesebezogenen (Ehm et al. 2011: $\eta^{2}=0,02$; Niklas und Schneider 2012: $d=0,27$ ) und mathematischen Selbstkonzepte (Ehm et al. 2011: $\eta^{2}=0,03$; Lindberg et al. 2013: $\eta^{2}=0,08$; Niklas und Schneider 2012: $d=0,27$ ) sowie die lesebezogene Lernfreude (Hellmich 2008: $d=0,26$ ). 


\subsection{Geschlechterstereotype als Erklärungsansatz domänenspezifischer Unterschiede von Mädchen und Jungen im Grundschulalter}

Einen zentralen Ansatz zur Erklärung der zuvor genannten Unterschiede bildet die Zuschreibung von Geschlechterstereotypen durch die Gesellschaft. Hierunter werden - folgt man Hannover und Wolter (2019) - Attribute verstanden, die Personen in Abhängigkeit ihres Geschlechts von den Mitgliedern einer Gesellschaft zugeschrieben werden. Im Detail bedeutet dies, dass Menschen klare stereotype (jedoch nicht zwangsläufig realitätsgetreue) Vorstellungen darüber haben, über welche grundlegenden Merkmale Männer und Frauen ihrer Meinung nach verfügen oder zumindest verfügen sollten (Hannover und Wolter 2019, S. 211). So werden Frauen beispielsweise häufig einfühlsame und rücksichtsvolle Eigenschaften zugeschrieben, wohingegen Männern Zielstrebigkeit und Dominanz attestiert werden (vgl. Hannover und Wolter 2019, S. 202-203; vgl. Hannover et al. 2014, S. 154-155). Auch viele Berufe werden als weibliche (z. B. Grundschullehrkraft) oder männliche Domänen (z. B. Pilot) angesehen (vgl. Cejka und Eagly 1999; Hannover 2008, S. 366).

Eine mögliche Erklärung für die geschlechtsspezifischen Unterschiede von Kindern im Grundschulalter stellt der lerntheoretische Ansatz dar. Demnach erlernen Mädchen und Jungen geschlechtsspezifische Stereotype bereits früh durch die Interaktion mit ihren Bezugspersonen (z. B. Eltern). Entspricht ein von Kindern gezeigtes Verhalten den durch die Gesellschaft geprägten (geschlechtsstereotypen) Vorstellungen der Bezugspersonen, werden sie in diesem Verhalten von ihnen bestärkt. Handeln sie entgegen diesen Vorannahmen, werden sie negativ sanktioniert. Während Bestärkungen die Wahrscheinlichkeit für ein erneutes Auftreten des gezeigten Verhaltens erhöhen, verringern negative Sanktionen diese. Den Kindern werden von ihren Bezugspersonen folglich Attribute vermittelt, die von der Gesellschaft als eher weibliche (z. B. Lesen) oder eher männliche Domänen (z. B. Mathematik) konnotiert werden (vgl. Hannover 2008).

Auch die stereotypen Geschlechtszuschreibungen von Lehrkräften im Grundschulunterricht könnten einen Faktor zur Erklärung von domänenspezifischen Unterschieden bei Grundschulkindern darstellen. Aktuell vorliegende Untersuchungen geben weitgehend einheitlich Hinweise darauf, dass Lehrerinnen und Lehrer Mädchen eine höhere Kompetenz im Lesen zuschreiben als Jungen (vgl. z. B. Boerma et al. 2016; Gentrup et al. 2018; Retelsdorf et al. 2015). Zusätzlich gaben die befragten Lehrerinnen und Lehrer in der Untersuchung von Gentrup et al. (2018) an, dass Mädchen im Vergleich zu Jungen motivierter im Lesen seien. Geschlechtsspezifische Vorannahmen von Lehrkräften zeigten sich vielfach auch dann, wenn Mädchen und Jungen in den einzelnen Domänen vergleichbare Leistungen aufwiesen (vgl. z. B. Keller 2001; Robinson-Cimpian et al. 2014; Tiedemann 2000). So zeigte sich beispielsweise in der Untersuchung von Robinson-Cimpian et al. (2014), dass die befragten Lehrkräfte die Kompetenzen der Jungen im Fach Mathematik höher einschätzten als diejenigen der Mädchen, obwohl gleiche Leistungen der Kinder vorlagen. Die Kompetenzen der Mädchen für das Fach Mathematik wurden nur dann als vergleichbar mit denjenigen der Jungen von den Lehrkräften wahrgenommen, wenn die Mädchen sich zusätzlich durch eine hohe Motivation auszeichneten. 
An dieser Stelle muss angemerkt werden, dass bestehende Geschlechterstereotype in der Gesellschaft selbstverständlich nicht den einzigen Ansatz zur Erklärung domänenspezifischer Unterschiede von Mädchen und Jungen im Grundschulalter darstellen (vgl. hierzu im Überblick: Hannover 2008). Da die weiteren Erklärungsansätze (z.B. genetische Unterschiede von Mädchen und Jungen) jedoch nicht im Fokus des vorliegenden Manuskripts standen, wurden sie hier nicht weiter ausgeführt.

\subsection{Die Folge geschlechtsstereotyper Vorstellungen von Lehrkräften in Hinblick auf ihr Feedbackverhalten gegenüber Mädchen und Jungen im Leseunterricht der Grundschule}

Unter Bezugnahme auf das Erwartungs-Mal-Wert-Modell von Eccles (2005) kann ein Zusammenhang zwischen den zuvor angeführten geschlechtsstereotypen Vorannahmen von Lehrkräften im Lesen (vgl. z.B. Boerma et al. 2016) und ihren Erwartungen an Schülerinnen und Schüler angenommen werden. Diese Erwartungshaltung der Lehrkräfte kann sich dann auf ihr konkretes Verhalten im Unterricht auswirken. Dabei muss es sich nicht zwangsläufig um realitätsgetreue Zuschreibungen seitens der Lehrkräfte handeln (vgl. Ludwig 2018; Rosenthal und Jacobsen 1971). Mit Blick auf den empirischen Forschungsstand konnten Good und Brophy (1978) als auch Weinstein et al. (1982) hierzu in ihren Untersuchungen belegen, dass die von der Lehrkraft als eher leistungsschwach eingeschätzten Schülerinnen und Schüler (im Vergleich zu den als leistungsstark angesehenen) tendenziell häufiger von der Lehrkraft in ihren Antworten unterbrochen werden. Des Weiteren wird ihnen weniger Zeit zum Beantworten von Fragen zur Verfügung gestellt. Bei richtigen Antworten werden sie seltener von der Lehrkraft gelobt, bei falschen Antworten häufiger kritisiert (vgl. hierzu auch im Überblick: Good et al. 2018). Zudem konnten Gentrup et al. (2020) Zusammenhänge zwischen der Einschätzung der Leistungen der Schülerinnen und Schüler durch die Lehrkräfte und ihrem Feedbackverhalten in den Fächern Mathematik und Deutsch belegen. Im Detail erteilten die Lehrkräfte mehr positives als negatives Feedback an die Schülerinnen und Schüler, wenn sie die Lernenden als leistungsstark einschätzten. Mit Blick auf den Leseunterricht der Grundschule kann auf der Grundlage der zuvor angeführten Inhalte folglich ein positiveres Feedbackverhalten der Lehrkräfte zugunsten der Mädchen angenommen werden.

Dabei wird unter Feedback eine Information verstanden, die Lehrerinnen und Lehrer Kindern zur Unterstützung ihrer Lernprozesse oder als Folge von Lernleistungen bereitstellen (vgl. Hattie 2009; Hattie und Timperley 2007). So kann positives Feedback von Lehrkräften dazu genutzt werden, die Leistungen von Schülerinnen und Schülern hervorzuheben (z.B. „Klasse!“). Mit der Erteilung von negativem Feedback wird das Ziel verfolgt, die Schülerinnen und Schüler darüber zu informieren, dass ihre Leistungen als generell verbesserungswürdig anzusehen sind (,Das hast du nicht gut gemacht!“; Pintrich und Schunk 2002, S. 326-327). Das Feedback von Lehrkräften stellt eine wichtige Determinante im Leseunterricht der Grundschule dar. Es hat die Funktion, Kinder in ihren Leseprozessen zu unterstützen (vgl. Neitzel und Davis 2014; Hattie 2009). Im Erstleseunterricht zu Beginn der Grund- 
schulzeit werden Kinder durch Feedback im Dekodieren und der Sinnerkennung von Wörtern gefördert (vgl. Schabmann et al. 2012). In den höheren Jahrgangsstufen der Grundschule (weiterführender Leseunterricht) stellen das laute Vorlesen sowie das individuelle (leise) Lesen von Texten die am häufigsten genutzten Unterrichtsformen dar (vgl. Stahns und Rieser 2018). Rückmeldungen der Lehrkraft werden dann verstärkt zur Unterstützung der Leseverständnisprozesse von Kindern genutzt (vgl. Schabmann et al. 2012).

Aktuell besteht ein defizitärer Forschungsstand zu der Fragestellung, ob sich die Erwartungen von Lehrkräften und ihr damit verbundenes Feedbackverhalten gegenüber Mädchen und Jungen in den einzelnen Jahrgangsstufen und den unterschiedlichen Formen des Leseunterrichts unterscheiden. Ebenso können gegenwärtig nur wenige empirische Untersuchungen angeführt werden, die sich mit Unterschieden in Bezug auf die Feedbackerteilung von Lehrkräften zu den Leistungen von Mädchen und Jungen im Grundschulunterricht auseinandergesetzt haben. Zudem muss angemerkt werden, dass viele der aktuell zur Thematik vorliegenden Untersuchungen (vgl. z. B. Irvine 1986; Simpson und Erickson 1983) schon vergleichsweise alt sind. Neuere Untersuchungen zu geschlechtsspezifischen Unterschieden in Bezug auf die Feedbackerteilung von Lehrkräften an Mädchen und Jungen im Grundschulalter liegen gegenwärtig mit wenigen Ausnahmen (vgl. z. B. Denn et al. 2015) kaum vor.

Im Kontext der gegenwärtig zur Thematik publizierten Forschungsbefunde berichten Simpson und Erickson (1983) in ihrer qualitativen Untersuchung über die Unterrichtsbeobachtung von 16 Grundschullehrkräften der ersten Klassenstufe. Sie kommen zu dem Schluss, dass die Lehrkräfte in ihren Klassen generell mehr negatives Feedback zu den Leistungen der Jungen erteilten. Keine geschlechtsspezifischen Unterschiede konnten dagegen bei der Erteilung positiven Feedbacks festgestellt werden. Zu vergleichbaren Ergebnissen in Bezug auf die Feedbackerteilung im Unterricht kommt auch Irvine (1986). Sie beobachtete die Interaktionen zwischen Lehrkräften und Kindern in 63 Klassenräumen aus 10 Vor- und Grundschulen. Auch die Ergebnisse der Untersuchung von Chen et al. (2011) decken sich mit den zuvor angeführten Forschungsbefunden. In ihrer quantitativen Querschnittsstudie befragten sie 1612 Schülerinnen und Schüler der dritten bis sechsten Klassenstufe zur Häufigkeit des von ihnen perzipierten positiven und negativen Feedbacks ihrer Lehrkräfte im Unterricht. Die Autoren konnten für die Wahrnehmung des positiven Feedbacks keine signifikanten Unterschiede zwischen den Schülerinnen und Schülern belegen. Allerdings zeigte sich beim perzipierten negativen Feedback ein signifikanter Unterschied mit mittlerer Effektstärke $\left(\eta^{2}=0,06\right)$ zwischen den beiden Gruppen. Demnach nehmen Jungen mehr negatives Feedback ihrer Lehrkräfte im Unterricht wahr als Mädchen.

In den zuvor genannten Untersuchungen wurden die geschlechtsspezifischen Unterschiede bei der Feedbackerteilung von Lehrkräften im Grundschulunterricht allgemein betrachtet. Dagegen gibt es aktuell kaum domänenspezifische Studien zur Thematik. So liegen speziell für den Leseunterricht der Grundschule gegenwärtig keine Untersuchungen vor. Allerdings kann für den Mathematikunterricht eine Studie von Denn et al. (2015) angeführt werden. In ihrer multiperspektivisch angelegten Untersuchung beobachteten die Autorinnen und Autoren 28 Klassen mit 465 Schülerinnen und Schülern der zweiten Jahrgangsstufe. Sie kommen zu dem Schluss, 
dass Jungen signifikant häufiger für ihr Verhalten getadelt werden als Mädchen. Dabei zeigte sich eine moderate Effektstärke $(d=0,36)$. Allerdings konnten keine geschlechtsspezifischen Unterschiede bei der Feedbackerteilung der Lehrkräfte zu den Leistungen der Kinder belegt werden.

\section{Forschungsdesiderata und Forschungshypothesen}

Mit Blick auf den empirischen Forschungsstand ist derzeit wenig darüber bekannt, inwiefern sich die Interaktion von Lehrkräften gegenüber Mädchen und Jungen in den einzelnen Schulfächern der Grundschule unterscheidet. In den Untersuchungen von Chen et al. (2011), Irvine (1986) sowie Simpson und Erickson (1983) zeigte sich einheitlich, dass Jungen häufiger negatives Feedback zu ihren Leistungen erhielten als Mädchen. Keine Unterschiede wurden dagegen in Bezug auf das positive Feedback deutlich. Befunde aus domänenspezifischen Untersuchungen liegen für die einzelnen Schulfächer aktuell nur vereinzelt vor. So konnten Denn et al. (2015) im Fach Mathematik keine Unterschiede zwischen Mädchen und Jungen in Bezug auf die Erteilung leistungsbezogenen Feedbacks belegen. Vergleichbare Untersuchungen für den Leseunterricht der Grundschule lassen sich mit Blick auf den aktuellen Forschungsstand nicht auffinden.

An dieses Forschungsdesiderat knüpft die vorliegende Untersuchung an. Dabei wird im Detail der Frage nachgegangen, ob Mädchen und Jungen ein unterschiedliches Feedbackverhalten ihrer Lehrkräfte im Leseunterricht der Grundschule wahrnehmen. Darüber hinaus wird untersucht, ob und inwiefern Unterschiede in dem von Grundschulkindern wahrgenommenen positiven und negativen Feedback ihrer Lehrkräfte über ihr Geschlecht, ihre Leistungen, ihre Motivation und ihre Selbstkonzepte erklärt werden können.

Vor dem Hintergrund einer besseren Leseleistung, einer stärker ausgeprägten Lesemotivation als auch höheren lesebezogenen Selbstkonzepten von Mädchen (vgl. Bos et al. 2012; Ehm et al. 2011; Hellmich 2008; Hornberg et al. 2007; Kessels und Heyder 2017; McElvany et al. 2017; McGeown 2015; McGeown et al. 2011; Niklas und Schneider 2012) wird die Annahme vertreten, dass sie im Vergleich zu Jungen eine häufigere Erteilung positiven Feedbacks im Leseunterricht der Grundschule wahrnehmen. Gleichzeitig kann anhand der zuvor genannten Befunde und auf der Grundlage der Ergebnisse der Untersuchungen von Chen et al. (2011), Gentrup et al. (2020), Good und Brophy (1978), Irvine (1986), Simpson und Erickson (1983) sowie Weinstein et al. (1982) angenommen werden, dass Jungen signifikant häufiger negatives Feedback ihrer Lehrkräfte im Leseunterricht wahrnehmen als Mädchen. Darüber hinaus weist eine Vielzahl an empirischen Studien darauf hin, dass Lehrkräfte geschlechtsstereotype Annahmen im Lesen vertreten (vgl. z. B. Boerma et al. 2016; Gentrup et al. 2018; Retelsdorf et al. 2015). Diese Stereotype konnten auch dann belegt werden, wenn Schülerinnen und Schüler über eine vergleichbare Leistung oder Motivation in den einzelnen Domänen verfügen (vgl. Keller 2001; Robinson-Cimpian et al. 2014; Tiedemann 2000). Aus diesem Grund wird zudem vermutet, dass Mädchen auch dann häufiger positives Feedback und seltener negatives Feedback ihrer Lehrkräfte im Leseunterricht der Grundschule wahrnehmen, 
wenn die Leistung, die Motivation sowie die Selbstkonzepte der Grundschulkinder kontrolliert werden.

Anhand der zuvor genannten empirischen Befunde können die nachfolgenden Forschungshypothesen abgeleitet werden:

$\mathbf{H}_{1 \mathrm{a}}$ Mädchen nehmen signifikant häufiger individuell an sie erteilte positive Rückmeldungen ihrer Lehrkräfte im Leseunterricht der Grundschule wahr als Jungen.

$\mathbf{H}_{1 \mathrm{~b}}$ Mädchen nehmen unter Kontrolle der Leseleistung, der Lesemotivation und der lesebezogenen Selbstkonzepte signifikant häufiger individuell an sie erteilte positive Rückmeldungen ihrer Lehrkräfte im Leseunterricht der Grundschule wahr als Jungen.

$\mathbf{H}_{2 a}$ Jungen nehmen signifikant häufiger individuell an sie erteilte negative Rückmeldungen ihrer Lehrkräfte im Leseunterricht der Grundschule wahr als Mädchen.

$\mathbf{H}_{2 \mathrm{~b}}$ Jungen nehmen unter Kontrolle der Leseleistung, der Lesemotivation und der lesebezogenen Selbstkonzepte signifikant häufiger individuell an sie erteilte negative Rückmeldungen ihrer Lehrkräfte im Leseunterricht der Grundschule wahr als Mädchen.

\section{Methode}

\subsection{Stichprobe}

An der vorliegenden Studie waren $N=665$ Schülerinnen und Schüler (341 Mädchen, 324 Jungen) der dritten und vierten Jahrgangsstufe aus 15 Schulen (46 Klassen) in Nordrhein-Westfalen beteiligt. Zum Zeitpunkt der Erhebung befanden sich 378 Kinder (186 Mädchen sowie 192 Jungen) in der dritten und 287 Schülerinnen und Schüler (155 Mädchen und 132 Jungen) in der vierten Jahrgangsstufe. Die Kinder der dritten Klassenstufe waren im Durchschnitt etwa 9 Jahre $(M=105,62$ Monate; $S D=5,63$ Monate; Min=77,11 Monate; $\operatorname{Max}=120,11$ Monate) und die Schülerinnen und Schüler der vierten Jahrgangsstufe etwa 10 Jahre alt $(M=120,96$ Monate; $S D=6,32$ Monate; $\operatorname{Min}=107,43$ Monate; $\operatorname{Max}=143,05$ Monate). Auf die Frage, welche Sprachen in der Familie erlernt wurden, gaben 614 Kinder $(92,3 \%)$ die deutsche Sprache an. Als weitere in der Familie erlernte Sprachen wurden in 46 Fällen Russisch $(6,9 \%)$, in 38 Fällen Türkisch $(5,7 \%)$, in 20 Fällen Italienisch $(3,0 \%)$ sowie in 13 Fällen Polnisch $(2,0 \%)$ von den Kindern genannt.

\subsection{Eingesetzte Messinstrumente}

Die an der Studie beteiligten Schülerinnen und Schüler beantworteten Fragen zu dem aus ihrer Sicht wahrgenommenen positiven und negativen Feedback ihrer Lehrkräfte im Leseunterricht der Grundschule, ihren lesebezogenen Selbstkonzepten sowie ihrer intrinsischen Lesemotivation und bearbeiteten den Leseverständnistest für Erst- bis 
Sechstklässler (ELFE 1-6; vgl. Lenhard und Schneider 2006). In Bezug auf die Antworten der Kinder zu den einzelnen Items zeigten sich 0,80\% fehlende Werte. Zur Vermeidung von Verzerrungen der Stichprobe wurden diese unter der Annahme, dass sie zufällig (,missing at random') fehlten, mit dem Expectation-MaximizationAlgorithmus (EM-Algorithmus) geschätzt (vgl. Leonhart 2009).

Zur Erfassung des aus Sicht der Kinder wahrgenommenen positiven und negativen Feedbacks ihrer Lehrkräfte wurden Skalen von Burnett (2002) genutzt. Diese wurden durch geschulte bilinguale Projektmitarbeiterinnen und -mitarbeiter aus dem Englischen übersetzt und geprüft. Für beide Skalen wurde eine fünfstufige LikertSkala $(1=$ nie, $2=$ eher nicht oft, $3=$ manchmal, $4=$ eher oft, $5=$ sehr oft $)$ zugrunde gelegt.

Die Fragebogenskala ,Wahrgenommenes positives Feedback der Lehrkräfte ' umfasste sechs Items $(M=3,38 ; S D=0,86 ; \operatorname{Min}=1,00 ; \operatorname{Max}=5,00 ; \alpha=0,86$; Beispiele: „Wie oft sagt dein Lehrer diese Sätze zu dir, nachdem du einen Text gelesen hast?“ - „Gut gemacht.“ oder „Du arbeitest wirklich gut mit.“). Ebenso wurde die Skala ,Wahrgenommenes negatives Feedback der Lehrkräfte“ über sechs Items erfasst $(M=1,77 ; S D=0,70 ; \operatorname{Min}=1,00 ; \operatorname{Max}=4,67 ; \alpha=0,81$; Beispiele: ,Wie oft sagt dein Lehrer diese Sätze zu dir, nachdem du einen Text gelesen hast?“ - „Das hast du nicht gut gelesen.“ oder „Du hast viele Fehler gemacht.“).

Die lesebezogenen Selbstkonzepte der Schülerinnen und Schüler wurden in Anlehnung an die Skalen zur Erfassung des schulischen Selbstkonzepts (SESSKO) von Schöne et al. (2002) erhoben, dabei allerdings leicht umformuliert. Der Skala lagen fünf Items zugrunde, die über ein fünfstufiges Differenzial erhoben wurden, bei dem nur die äußeren Pole beschriftet waren $(M=4,00 ; S D=0,75 ;$ Min =1,00; Max = 5,00; $\alpha=0,84$; Beispiele: ,Wenn ich mir angucke, was wir beim Lesen können müssen, glaube ich, dass ich wenig kann/viel kann.“ oder „Wenn ich mir angucke, was wir beim Lesen können müssen, finde ich, dass ich mit den Aufgaben in der Schule nicht gut zurechtkomme/gut zurechtkomme.").

Zusätzlich beantworteten die Schülerinnen und Schüler Fragen zu ihrer intrinsischen Lesemotivation. Hierbei wurde ein vierstufiges Antwortformat zugrunde gelegt $(1=$ nein, $2=$ eher nein, $3=$ eher ja, $4=$ ja). Die in diesem Zusammenhang genutzten Items wurden von Stutz et al. (2017) entwickelt (4 Items; $M=3,34 ; S D=0,73$; Min = 1,00; Max=4,00; $\alpha=0,81$; Beispiel: ,Warum liest du, wenn du nicht in der Schule bist?“ - „Ich lese, weil es Spaß macht.“ oder „Ich lese, weil es aufregend ist, was den Personen in einer Geschichte so passiert.").

Darüber hinaus bearbeiteten die Schülerinnen und Schüler den Subtest ,Textverständnis ' aus dem Leseverständnistest ELFE (Lenhard und Schneider 2006). Bei der Skala wurde eine dichotome Codierung vorgenommen $(0=$ falsch/nicht beantwortet; 1 = richtig). Die 20 eingesetzten Items wurden anschließend zu einem Summenwert aufaddiert $(M=12,78 ; S D=5,08 ;$ Min $=0,00 ; \operatorname{Max}=20,00)$.

\subsection{Durchführung}

Die Erhebung der Daten erfolgte in einer Unterrichtsstunde (45 Minuten) im regulären Schulalltag durch mehrere geschulte Projektmitarbeiterinnen und -mitarbeiter. Dabei wurden die einzelnen Fragebogenitems von den zuständigen Erhebungslei- 
terinnen und -leitern laut im Klassenverband vorgelesen, so dass alle Kinder den einzelnen Aussagen im Fragebogen folgen und zu diesen Stellung nehmen konnten.

\subsection{Ergebnisse}

Im Folgenden werden zunächst die deskriptiven Befunde zu den einzelnen Variablen (wahrgenommenes Feedback, Selbstkonzept, intrinsische Motivation und Leseleistung) aufgeführt. Dabei werden zudem anhand von t-tests Mittelwertsunterschiede zwischen Mädchen und Jungen berichtet (siehe Tab. 1).

Es wird ersichtlich, dass Mädchen (im Vergleich zu den Jungen) eine stärker ausgeprägte intrinsische Motivation sowie ein höheres Leseverständnis aufweisen. In Hinblick auf die lesebezogenen Selbstkonzepte zeigen sich dagegen keine signifikanten geschlechtsspezifischen Unterschiede. Die signifikanten Unterschiede von Mädchen und Jungen im Grundschulalter weisen dabei kleine Effektstärken auf (vgl. Cohen 1992).

Nachfolgend werden die Ergebnisse aus zwei univariaten zwei-faktoriellen Kovarianzanalysen mit dem von den Kindern perzipierten positiven und negativen Feedback als abhängige Variablen präsentiert. Sie dienen zur Überprüfung der Hypothesen $1_{\mathrm{a}}, 1_{\mathrm{b}}, 2_{\mathrm{a}}$ und $2_{\mathrm{b}}$. Einen Faktor bildet dabei das Geschlecht der Kinder $(0=$ Mädchen, $1=$ Junge $)$. Zusätzlich wird der Faktor der Leistung $(0=$ eher leistungsschwach, $1=$ eher leistungsstark) mit in die Analysen einbezogen. Die Einteilung der Schülerinnen und Schüler in eine eher leistungsschwache und eine eher leistungsstarke Gruppe erfolgte unter Bezugnahme auf die Normwerte der Eichstichprobe des Subtests ,Textverständnis ‘ des Inventars ,Ein Leseverständnistest für Erstbis Sechstklässler' (ELFE 1-6). Als Cut-Off-Kriterium dienten dabei jeweils die Punktzahlen für Dritt- und Viertklässler zum Schuljahresende, die zur Erreichung des Prozentrangs 50 (durchschnittliche Leistung) der jeweiligen Jahrgangsstufe der Eichstichprobe notwendig waren. Im Detail entsprach dies 16 Punkten für Kinder

Tab. 1 Geschlechtsspezifische Unterschiede in Bezug auf die eingesetzten Variablen

\begin{tabular}{|c|c|c|c|c|c|c|c|c|}
\hline & \multicolumn{2}{|c|}{$\begin{array}{l}\text { Mädchen } \\
(N=341)\end{array}$} & \multicolumn{2}{|c|}{$\begin{array}{l}\text { Jungen } \\
(N=324)\end{array}$} & \multicolumn{4}{|c|}{$\begin{array}{l}\text { Signifikanztest der Mittelwertsunterschiede } \\
\text { (t-Tests) }\end{array}$} \\
\hline & $M$ & $S D$ & $M$ & $S D$ & $t$ & $D f$ & $p$ & $d$ \\
\hline $\begin{array}{l}\text { Wahrgenommenes } \\
\text { positives } \\
\text { Feedback }^{\mathrm{a}}\end{array}$ & 3,46 & 0,85 & 3,30 & 0,87 & $-2,40$ & 663 & $\leq 0,05$ & 0,19 \\
\hline $\begin{array}{l}\text { Wahrgenommenes } \\
\text { negatives } \\
\text { Feedback }^{\mathrm{a}}\end{array}$ & 1,68 & 0,69 & 1,85 & 0,71 & 3,23 & 663 & $\leq 0,001$ & 0,25 \\
\hline $\begin{array}{l}\text { Lesebezogenes } \\
\text { Selbstkonzept }\end{array}$ & 4,02 & 0,72 & 3,99 & 0,77 & 0,58 & 663 & 0,56 & 0,05 \\
\hline $\begin{array}{l}\text { Intrinsische } \\
\text { Lesemotivation }^{\mathrm{c}}\end{array}$ & 3,48 & 0,60 & 3,19 & 0,82 & $-5,26$ & 589,15 & $\leq 0,001$ & 0,41 \\
\hline Leseverständnis $^{\mathrm{d}}$ & 13,72 & 4,83 & 11,80 & 5,16 & $-4,96$ & 663 & $\leq 0,001$ & 0,39 \\
\hline
\end{tabular}

${ }^{\mathrm{a}} 1$ = nie, $2=$ eher nicht oft, $3=$ manchmal, $4=$ eher oft, $5=$ sehr oft

bünfstufiges Differenzial, bei dem nur die äußeren Pole beschriftet waren

${ }^{\mathrm{c}} 1=$ nein, $2=$ eher nein, $3=$ eher ja, $4=$ ja

${ }^{\mathrm{d}} 0=$ falsch/nicht beantwortet, $1=$ richtig (Summenwert der Items) 
Tab. 2 Geschlechtsspezifische Unterschiede leistungsschwächerer und leistungsstärkerer Kinder in Bezug auf das von ihnen perzipierte positive und negative Feedback

\begin{tabular}{|c|c|c|c|c|c|c|c|c|}
\hline & $M$ & $S D$ & $M$ & $S D$ & $t$ & $D f$ & $p$ & $d$ \\
\hline $\begin{array}{l}\text { Leistungs- } \\
\text { schwächere }\end{array}$ & \multicolumn{2}{|c|}{$\begin{array}{l}\text { Mädchen } \\
(N=147)\end{array}$} & \multicolumn{2}{|c|}{$\begin{array}{l}\text { Jungen } \\
(N=197)\end{array}$} & \multicolumn{4}{|c|}{$\begin{array}{l}\text { Signifikanztest der Mittelwertsunterschiede } \\
(\text { t-Tests })\end{array}$} \\
\hline $\begin{array}{l}\text { Perzipiertes } \\
\text { positives } \\
\text { Feedback }^{\mathrm{a}}\end{array}$ & 3,33 & 0,81 & 3,16 & 0,84 & $-1,88$ & 342 & 0,06 & 0,21 \\
\hline $\begin{array}{l}\text { Perzipiertes } \\
\text { negatives } \\
\text { Feedback }^{\mathrm{a}}\end{array}$ & 1,91 & 0,79 & 2,01 & 0,75 & 1,19 & 342 & 0,24 & 0,13 \\
\hline Leistungsstärkere & \multicolumn{2}{|c|}{$\begin{array}{l}\text { Mädchen } \\
(N=194)\end{array}$} & \multicolumn{2}{|c|}{$\begin{array}{l}\text { Jungen } \\
(N=127)\end{array}$} & \multicolumn{4}{|c|}{$\begin{array}{l}\text { Signifikanztest der Mittelwertsunterschiede } \\
(t \text {-Tests })\end{array}$} \\
\hline $\begin{array}{l}\text { Perzipiertes } \\
\text { positives } \\
\text { Feedback }^{\mathrm{a}}\end{array}$ & 3,56 & 0,87 & 3,52 & 0,87 & $-0,42$ & 319 & 0,68 & 0,05 \\
\hline $\begin{array}{l}\text { Perzipiertes } \\
\text { negatives } \\
\text { Feedback }^{\mathrm{a}}\end{array}$ & 1,51 & 0,54 & 1,62 & 0,59 & 1,78 & 319 & 0,08 & 0,20 \\
\hline
\end{tabular}

Die Kinder wurden anhand der Normwerte der Eichstichprobe ihrer jeweiligen Jahrgangsstufe in eine leistungsschwächere (unterhalb Prozentrang 50) und eine leistungsstärkere Gruppe (Prozentrang 50 und höher) eingeteilt (vgl. Lenhard und Schneider 2006, S. 57-59)

${ }^{\mathrm{a}} 1$ = nie, $2=$ eher nicht oft, $3=$ manchmal, $4=$ eher oft, $5=$ sehr oft

der vierten sowie 13 Punkten für Schülerinnen und Schüler der dritten Klassenstufe (vgl. Lenhard und Schneider 2006, S. 57-59). In Tab. 2 finden sich die deskriptiven Werte sowie Gruppenvergleiche zu den gebildeten Leistungsgruppen und dem jeweils wahrgenommenen Feedback.

Die Berechnung der Effekte der univariaten Kovarianzanalysen erfolgt im Folgenden jeweils in vier verschiedenen Modellen. Zunächst wird lediglich der Faktor Geschlecht in die Analysen integriert, um zu überprüfen, ob Unterschiede in dem von Kindern perzipierten positiven und negativen Feedback über ihr Geschlecht erklärt werden können. In Modell 2 wird die Analyse um den Faktor Leistung und den Interaktionseffekt der beiden Variablen (,Geschlecht $\times$ Leistung') ergänzt. In den Modellen 3 und 4 werden dann schrittweise die Kovariaten ,intrinsische Lesemotivation ' und ,lesebezogenes Selbstkonzept' in die Analysen eingeführt. Die Ergebnisse der berechneten Kovarianzanalyse zu dem von Kindern im Leseunterricht wahrgenommenen positiven Feedback können der nachfolgenden Tab. 3 entnommen werden.

Wie anhand der durchgeführten univariaten Kovarianzanalyse zum wahrgenommenen positiven Feedback deutlich wird, ergibt sich in Modell 1 zunächst ein signifikanter Haupteffekt des Geschlechts. Demnach nehmen Mädchen im Leseunterricht der Grundschule signifikant mehr positives Feedback ihrer Lehrkräfte wahr als Jungen. Folglich kann Hypothese $1_{\mathrm{a}}$ als bestätigt angesehen werden. Allerdings muss angemerkt werden, dass lediglich eine kleine Effektstärke vorliegt. Wird in Modell 2 die Leistung der Kinder als zusätzlicher Faktor mit in die Analysen einbezogen, kann kein signifikanter Haupteffekt des Geschlechts mehr belegt werden. Ebenso zeigt sich kein signifikanter Interaktionseffekt (Geschlecht $\times$ Leistung). Da- 
Tab. 3 Kovarianzanalyse zu dem von Kindern wahrgenommenen positiven Feedback ihrer Lehrkräfte

\begin{tabular}{|c|c|c|c|c|}
\hline & $\begin{array}{l}\text { Modell } 1 \\
R^{2}=0,01\end{array}$ & $\begin{array}{l}\text { Modell } 2 \\
R^{2}=0,04\end{array}$ & $\begin{array}{l}\text { Modell } 3 \\
R^{2}=0,06\end{array}$ & $\begin{array}{l}\text { Modell } 4 \\
R^{2}=0,20\end{array}$ \\
\hline $\begin{array}{l}\text { Haupteffekt A } \\
\text { (Geschlecht) }\end{array}$ & $\begin{array}{l}F=5,74 \\
p \leq \mathbf{0 , 0 5} \\
\eta_{p}^{2}=0,01\end{array}$ & $\begin{array}{l}F=2,49 \\
p=0,12 \\
\eta_{p}^{2}=0,00\end{array}$ & $\begin{array}{l}F=0,66 \\
p=0,42 \\
\eta_{p}^{2}=0,00\end{array}$ & $\begin{array}{l}F=2,27 \\
p=0,13 \\
\eta_{p}^{2}=0,00\end{array}$ \\
\hline $\begin{array}{l}\text { Haupteffekt B } \\
\text { (Leistung) }\end{array}$ & - & $\begin{array}{l}F=18,96 \\
\boldsymbol{p} \leq \mathbf{0 , 0 0 1} \\
\eta_{p}^{2}=0,03\end{array}$ & $\begin{array}{l}F=13,14 \\
\boldsymbol{p} \leq \mathbf{0 , 0 0 1} \\
\eta_{p}^{2}=0,02\end{array}$ & $\begin{array}{l}F=2,65 \\
p=0,10 \\
\eta_{p}^{2}=0,00\end{array}$ \\
\hline $\begin{array}{l}\text { Interaktion } \\
(\text { Geschlecht } \times \text { Leistung })\end{array}$ & - & $\begin{array}{l}F=0,92 \\
p=0,34 \\
\eta_{p}^{2}=0,00\end{array}$ & $\begin{array}{l}F=1,39 \\
p=0,24 \\
\eta_{p}^{2}=0,00\end{array}$ & $\begin{array}{l}F=1,47 \\
p=0,23 \\
\eta_{p}^{2}=0,00\end{array}$ \\
\hline $\begin{array}{l}\text { Kovariate A } \\
\text { (Intrinsische Lese- } \\
\text { motivation) }\end{array}$ & - & - & $\begin{array}{l}F=19,06 \\
p \leq \mathbf{0 , 0 0 1} \\
\eta_{p}^{2}=0,03\end{array}$ & $\begin{array}{l}F=5,34 \\
p \leq \mathbf{0 , 0 5} \\
\eta_{p}^{2}=0,01\end{array}$ \\
\hline $\begin{array}{l}\text { Kovariate B } \\
\text { (Lesebezogenes } \\
\text { Selbstkonzept) }\end{array}$ & - & - & - & $\begin{array}{l}F=110,55 \\
\boldsymbol{p} \leq \mathbf{0 , 0 0 1} \\
\eta_{p}^{2}=0,14\end{array}$ \\
\hline
\end{tabular}

Die Kinder wurden anhand der Normwerte der Eichstichprobe ihrer jeweiligen Jahrgangsstufe in eine leistungsschwächere (unterhalb Prozentrang 50) und eine leistungsstärkere Gruppe (Prozentrang 50 und höher) eingeteilt (vgl. Lenhard und Schneider 2006, S. 57-59)

gegen wird ein signifikanter Haupteffekt für die Leistungen der Schülerinnen und Schüler mit kleiner Effektstärke deutlich. Durch Einführung der Kovariate ,intrinsische Motivation“ in Modell 3 zeigen sich signifikante kleine Effekte für den Faktor ,Leistung' sowie für die Kovariate ,intrinsische Motivation“. Unter Einbezug der lesebezogenen Selbstkonzepte der Kinder in Modell 4 werden nur noch signifikante Effekte für die beiden Kovariaten sichtbar. Dabei wird eine kleine Effektstärke für die intrinsische Motivation sowie eine große Effektstärke für das lesebezogene Selbstkonzept evident. In Hinblick auf das Geschlecht der Kinder zeigen sich in keinem der Modelle 2-4 signifikante Ergebnisse. Folglich muss Hypothese $1_{b}$ abgelehnt werden.

Ebenso werden im Folgenden vergleichbare Analysen zu dem von Kindern wahrgenommenen negativen Feedback ihrer Lehrkräfte im Leseunterricht der Grundschule vorgenommen. Die Ergebnisse der berechneten Kovarianzanalyse mit dem perzipierten negativen Feedback als abhängige Variable lassen sich der Tab. 4 entnehmen.

Aus Modell 1 wird ein signifikanter Haupteffekt des Geschlechts ersichtlich. Somit nehmen Jungen signifikant mehr negatives Feedback ihrer Lehrkräfte im Leseunterricht der Grundschule wahr als Mädchen. Dementsprechend kann Hypothese 2a angenommen werden. Angemerkt werden muss jedoch, dass sich der berichtete Effekt als klein erweist.

Auch unter Einbezug des Faktors ,Leistung' in Modell 2 zeigt sich ein signifikanter Haupteffekt des Geschlechts. Allerdings verringert sich die Effektstärke der Variable ,Geschlecht' durch den Einbezug des Faktors ,Leistung', für den ein signifikanter Haupteffekt belegt werden kann. Im Detail wird in Modell 2 ein kleiner Effekt für das Geschlecht und ein mittlerer Effekt für die Leistung der Kinder ersichtlich. Dagegen kann kein signifikanter Interaktionseffekt (Geschlecht $\times$ Leistung) 
Tab. 4 Kovarianzanalyse zu dem von Kindern wahrgenommenen negativen Feedback ihrer Lehrkräfte

\begin{tabular}{|c|c|c|c|c|}
\hline & $\begin{array}{l}\text { Modell } 1 \\
R^{2}=0,02\end{array}$ & $\begin{array}{l}\text { Modell } 2 \\
R^{2}=0,09\end{array}$ & $\begin{array}{l}\text { Modell } 3 \\
R^{2}=0,12\end{array}$ & $\begin{array}{l}\text { Modell } 4 \\
R^{2}=0,15\end{array}$ \\
\hline $\begin{array}{l}\text { Haupteffekt A } \\
\text { (Geschlecht) }\end{array}$ & $\begin{array}{l}F=10,41 \\
p \leq \mathbf{0 , 0 0 1} \\
\eta_{p}^{2}=0,02\end{array}$ & $\begin{array}{l}F=4,00 \\
p \leq \mathbf{0 , 0 5} \\
\eta_{p}^{2}=0,01\end{array}$ & $\begin{array}{l}F=1,32 \\
p=0,25 \\
\eta_{p}^{2}=0,00\end{array}$ & $\begin{array}{l}F=2,03 \\
p=0,16 \\
\eta_{p}^{2}=0,00\end{array}$ \\
\hline $\begin{array}{l}\text { Haupteffekt B } \\
\text { (Leistung) }\end{array}$ & - & $\begin{array}{l}F=53,92 \\
\boldsymbol{p} \leq \mathbf{0 , 0 0 1} \\
\eta_{p}^{2}=0,08\end{array}$ & $\begin{array}{l}F=42,90 \\
p \leq \mathbf{0 , 0 0 1} \\
\eta_{p}^{2}=0,06\end{array}$ & $\begin{array}{l}F=31,04 \\
p \leq \mathbf{0 , 0 0 1} \\
\eta_{p}^{2}=0,05\end{array}$ \\
\hline $\begin{array}{l}\text { Interaktion } \\
(\text { Geschlecht } \times \text { Leistung })\end{array}$ & - & $\begin{array}{l}F=0,02 \\
p=89 \\
\eta_{p}^{2}=0,00\end{array}$ & $\begin{array}{l}F=0,01 \\
p=0,92 \\
\eta_{p}^{2}=0,00\end{array}$ & $\begin{array}{l}F=0,01 \\
p=0,94 \\
\eta_{p}^{2}=0,00\end{array}$ \\
\hline $\begin{array}{l}\text { Kovariate A } \\
\text { (Intrinsische Lese- } \\
\text { motivation) }\end{array}$ & - & - & $\begin{array}{l}F=24,06 \\
p \leq \mathbf{0 , 0 0 1} \\
\eta_{p}^{2}=0,04\end{array}$ & $\begin{array}{l}F=15,17 \\
p \leq \mathbf{0 , 0 0 1} \\
\eta_{p}^{2}=0,02\end{array}$ \\
\hline $\begin{array}{l}\text { Kovariate B } \\
\text { (Lesebezogenes } \\
\text { Selbstkonzept) }\end{array}$ & - & - & - & $\begin{array}{c}F=19,37 ; \\
\quad \boldsymbol{p} \leq \mathbf{0 , 0 0 1} ; \\
\eta_{p}^{2}=0,03\end{array}$ \\
\hline
\end{tabular}

Die Kinder wurden anhand der Normwerte der Eichstichprobe ihrer jeweiligen Jahrgangsstufe in eine leistungsschwächere (unterhalb Prozentrang 50) und eine leistungsstärkere Gruppe (Prozentrang 50 und höher) eingeteilt (vgl. Lenhard und Schneider 2006, S. 57-59)

belegt werden. Auch in Modell 3 kann ein signifikanter Haupteffekt für die Leistung mit mittlerer Effektstärke aufgezeigt werden. Ebenso wird ein signifikanter Effekt für die Kovariate ,intrinsische Motivation ‘ ersichtlich. Dieser weist eine kleine Effektstärke auf. Der Haupteffekt für das Geschlecht sowie der Interaktionseffekt verfehlen das signifikante Niveau dagegen. Vergleichbare Effekte zeigen sich auch in Modell 4, in dem die beiden Kovariaten ,intrinsische Motivation ' und ,lesebezogenes Selbstkonzept‘ signifikante Prädiktoren zur Erklärung von Unterschieden im wahrgenommenen negativen Feedback von Kindern im Grundschulalter ausbilden. Zudem erweist sich der Haupteffekt für den Faktor ,Leistung ' als signifikant. Für alle drei Effekte können kleine Effektstärken angeführt werden. Geschlechtsspezifische Effekte werden in Modell 4 nicht evident. Zusammenfassend zeigen die Ergebnisse der durchgeführten Kovarianzanalyse, dass das von Kindern perzipierte negative Feedback im Leseunterricht der Grundschule in Modell 2 auch unter Kontrolle der Leistung über den Faktor, Geschlecht' erklärt werden kann. In den Modellen 3 und 4 kann das von Kindern wahrgenommene negative Feedback ihrer Lehrkräfte signifikant über ihre intrinsische Motivation, ihre lesebezogenen Selbstkonzepte als auch ihre Leseleistung erklärt werden, nicht aber über ihr Geschlecht. Hypothese $2_{b}$ muss aus diesem Grund abgelehnt werden. Es ergeben sich jedoch differenzierte Effekte, die in der Diskussion aufgegriffen werden.

\section{Diskussion}

Im Rahmen der vorliegenden Untersuchung wurden zwei zentrale Fragestellungen bearbeitet. Einerseits wurde der Frage nachgegangen, ob sich die Wahrnehmung des von Lehrkräften erteilten positiven und negativen Feedbacks aus der Sicht von 
Mädchen und Jungen im Leseunterricht der Grundschule unterscheidet. Andererseits wurde untersucht, ob und inwiefern Unterschiede in dem von Grundschulkindern wahrgenommenen positiven und negativen Feedback ihrer Lehrkräfte im Lesen über ihr Geschlecht, ihre Leistungen, ihre Motivation und ihre Selbstkonzepte erklärt werden können. Den Ausgangspunkt für die Bearbeitung dieser Thematik bildeten die in zahlreichen Untersuchungen aufgezeigten lesebezogenen Unterschiede von Mädchen und Jungen im Grundschulalter (vgl. z. B. Bos et al. 2012). Als ein Ansatz zur Erklärung der zuvor genannten Unterschiede wurden die von Lehrkräften vertretenen Vorannahmen im Lesen angeführt (vgl. z. B. Boerma et al. 2016). Im Detail wurde angenommen, dass Lehrerinnen und Lehrer an Grundschulen aufgrund der von ihnen im Lesen vermuteten Geschlechterstereotype niedrigere Erwartungen an Jungen (im Vergleich zu Mädchen) im Leseunterricht stellen würden (vgl. z.B. Ludwig 2018; Rosenthal und Jacobsen 1971). Diese Erwartungshaltungen von Lehrkräften könnten dann zu einer unterschiedlich häufigen Erteilung positiven und negativen Feedbacks an Mädchen und Jungen im Leseunterricht der Grundschule führen, welche von Grundschulkindern als solche wahrgenommen wird (vgl. Gentrup et al. 2020; Good und Brophy 1978; Good et al. 2018; Weinstein et al. 1982).

Anhand der Ergebnisse einer durchgeführten Kovarianzanalyse kann in Hinblick auf die Wahrnehmung des positiven Feedbacks erwartungskonform belegt werden, dass Mädchen im Vergleich zu Jungen eine signifikant häufigere Erteilung positiven Feedbacks ihrer Lehrkräfte im Leseunterricht der Grundschule perzipieren. Entgegen den Erwartungen konnte unter Kontrolle der Leseleistung, der intrinsischen Lesemotivation und der lesebezogenen Selbstkonzepte der Kinder allerdings kein geschlechtsspezifischer Effekt belegt werden. Ebenso wurde in keinem der berechneten Modelle ein signifikanter Interaktionseffekt (Geschlecht $\times$ Leistung) in Bezug auf das von Kindern wahrgenommene positive Feedback ihrer Lehrkräfte evident. Die Befunde geben damit Hinweise darauf, dass das von Kindern perzipierte positive Feedback stärker über ihre Leistungen, ihre Motivation sowie ihre Selbstkonzepte im Lesen (vgl. Bos et al. 2012; Ehm et al. 2011; Hellmich 2008; Hornberg et al. 2007; Kessels und Heyder 2017; McElvany et al. 2017; McGeown 2015; McGeown et al. 2011; Niklas und Schneider 2012) und weniger über ihr Geschlecht erklärt werden kann. Demnach spielt das Geschlecht der Kinder offenbar eine eher untergeordnete Rolle hinsichtlich ihrer Wahrnehmung des erteilten positiven Feedbacks ihrer Lehrkräfte im Leseunterricht der Grundschule.

Die Ergebnisse zu dem von Kindern im Leseunterricht wahrgenommenen negativen Feedback ihrer Lehrkräfte zeigen ebenso erwartungskonform, dass Jungen eine signifikant häufigere Erteilung negativen Feedbacks wahrnehmen als Mädchen. Der Befund geht somit mit denjenigen aus den Studien von Chen et al. (2011), Irvine (1986) sowie Simpson und Erickson (1983) einher. In den angeführten Untersuchungen konnte einheitlich eine häufigere Erteilung negativen Feedbacks der Lehrkräfte an Jungen (im Vergleich zu den Mädchen) belegt werden. Der signifikante Haupteffekt des Geschlechts blieb auch dann erhalten, wenn die Leseleistungen der Schülerinnen und Schüler kontrolliert wurden. Dementsprechend nehmen Jungen (im Vergleich zu Mädchen) auch unter Kontrolle der Leseleistung eine häufigere Erteilung negativen Feedbacks ihrer Lehrkräfte im Leseunterricht der Grundschule wahr. Einen möglichen Erklärungsansatz für dieses Ergebnis könnten Tendenzen 
von Lehrkräften zu geschlechtsspezifischen Vorannahmen im Lesen bilden (vgl. hierzu auch Boerma et al. 2016; Gentrup et al. 2018; Keller 2001; Retelsdorf et al. 2015; Robinson-Cimpian et al. 2014; Tiedemann 2000). So wäre denkbar, dass von Lehrkräften vertretene Geschlechterstereotype dazu führen könnten, dass diese eine niedrigere Erwartungshaltung gegenüber Jungen im Lesen vertreten (vgl. Gentrup et al. 2020; Good und Brophy 1978; Ludwig 2018; Rosenthal und Jacobsen 1971; Weinstein et al. 1982), infolgedessen sie häufiger negatives Feedback an Jungen im Leseunterricht der Grundschule erteilen.

Werden in einem weiteren Schritt dann allerdings die intrinsische Motivation sowie die Selbstkonzepte der Kinder im Lesen kontrolliert, erweist sich ihr Geschlecht nicht mehr als signifikanter Prädiktor zur Erklärung des von ihnen perzipierten negativen Feedbacks der Lehrkräfte. Diese Befunde könnten dahingehend gedeutet werden, dass der Leistung, der Motivation und den Selbstkonzepten der Kinder eine höhere Bedeutsamkeit hinsichtlich des perzipierten negativen Feedbacks ihrer Lehrkräfte zugesprochen werden kann als ihrem Geschlecht. So sinkt die Effektstärke des Haupteffekts für das Geschlecht bereits durch die Kontrolle der Leistung, obschon er noch ein signifikantes Niveau aufweist. Durch den weiteren Einbezug der intrinsischen Motivation als Kovariate verschwindet dieser geringe Effekt. Zudem zeigen sich (im Vergleich zum Faktor ,Geschlecht ${ }^{\circ}$ ) in allen Modellen höhere Effektstärken der Leistung, der Motivation und der Selbstkonzepte der Kinder. Möglicherweise könnten die Ergebnisse der vorgestellten Untersuchung über einen indirekten Vorhersagewert des Geschlechts der Kinder hinsichtlich ihrer Wahrnehmung des negativen Feedbacks ihrer Lehrkräfte erklärt werden: Mädchen und Jungen unterscheiden sich demnach durch gesellschaftlich geprägtes und erlerntes geschlechtsspezifisches Verhalten voneinander. Im Detail zeigen Jungen (im Vergleich zu Mädchen) im Lesen niedrigere Leistungen, eine geringere Motivation sowie schwächer ausgeprägte Selbstkonzepte (vgl. z. B. Hannover 2008; Ehm et al. 2011; McElvany et al. 2017) und nehmen aus diesem Grund vermutlich auch mehr negatives Feedback ihrer Lehrkräfte im Unterricht wahr. Folglich würden Unterschiede im wahrgenommenen negativen Feedback der Kinder nicht direkt über ihr Geschlecht erklärt werden, sondern über hiermit zusammenhängende geschlechtsspezifische Verhaltensweisen (vgl. Hannover 2008). Der konkrete Befund, dass erst unter Kontrolle der Motivation (und Selbstkonzepte) der Kinder kein geschlechtsspezifischer Effekt zur Erklärung ihres wahrgenommenen negativen Feedbacks mehr belegt werden kann, könnte unter Bezugnahme auf das Erwartungs-mal-Wert-Modell von Eccles (2005) darüber erklärt werden, dass die Feedbackerteilung von Lehrkräften im Unterricht einen komplexen Sachverhalt darstellt, der von einer Vielzahl an Faktoren abhängig ist. Somit kann das perzipierte negative Feedback der Schülerinnen und Schüler womöglich nicht allein über ihre Leistung erklärt werden. Vermutlich spielt auch das konkrete Verhalten der Schülerinnen und Schüler (beispielsweise in Form von motiviertem Handeln) eine nicht zu vernachlässigende Rolle hinsichtlich der Feedbackerteilung von Lehrkräften im Unterricht (vgl. Robinson-Cimpian et al. 2014).

Resümierend geben die Befunde der vorliegenden Untersuchung somit Hinweise darauf, dass sich die aufgefundenen geschlechtsspezifischen Unterschiede in dem von Kindern wahrgenommenen positiven und negativen Feedback ihrer Lehrkräfte im Leseunterricht der Grundschule stärker über leistungsbezogene Variablen als 
über ihr Geschlecht erklären lassen. Mädchen nehmen demnach vermutlich mehr positives und weniger negatives Feedback im Leseunterricht der Grundschule wahr, da sie (im Vergleich zu Jungen) tendenziell über bessere Leseleistungen (vgl. z. B. Bos et al. 2012; Ehm et al. 2011; Hellmich 2008; Hornberg et al. 2007; Kessels und Heyder 2017; McElvany et al. 2017; Niklas und Schneider 2012), eine höhere Lesemotivation sowie stärker ausgeprägte lesebezogene Selbstkonzepte (vgl. z. B. Ehm et al. 2011; Hornberg et al. 2007; McElvany et al. 2017; McGeown 2015; McGeown et al. 2011; Niklas und Schneider 2012) verfügen.

Über die Diskussion der Befunde hinaus soll im Folgenden auf einige Limitationen der vorgestellten Untersuchung aufmerksam gemacht werden. Hinsichtlich der eingesetzten Skalen zur Erfassung des von Kindern perzipierten positiven und negativen Feedbacks muss angemerkt werden, dass die Übersetzung der englischen Originalskala ins Deutsche nicht statistisch überprüft wurde. Im Rahmen weiterer Untersuchungen sollte die Übersetzung anhand einer englisch- und einer deutschsprachigen Stichprobe auf vorliegende Messinvarianz getestet werden. Ebenso sollte der Fragestellung nachgegangen werden, wie gut die eingesetzten Fragebogenitems realistisches Feedback von Lehrkräften im Leseunterricht der Grundschule abbilden. Möglicherweise könnten die Antworten der Schülerinnen und Schüler zu dem von ihnen perzipierten positiven und negativen Feedback ihrer Lehrkräfte dadurch verzerrt worden sein, dass die ihnen vorgelegten Itemaussagen in dieser Form eher selten im Lesen erteilt wurden.

Diskutiert werden sollte in diesem Zusammenhang auch, dass in der vorliegenden Untersuchung (lediglich) das von Kindern perzipierte positive und negative Feedback im Lesen erfasst wurde. Dabei lag die Annahme zugrunde, dass das von Lehrkräften im Leseunterricht erteilte positive und negative Feedback realitätsgetreu von den Schülerinnen und Schülern perzipiert wurde. Obwohl die Untersuchung von Glen et al. (2004) Hinweise darauf liefert, dass Zusammenhänge zwischen dem von Lehrkräften im Unterricht erteilten Feedback und der Wahrnehmung der von ihnen erteilten Rückmeldungen durch Schülerinnen und Schüler vorliegen, ist diese Thematik in der empirischen Lehr-Lern-Forschung gegenwärtig kaum untersucht. Zudem wäre es möglich, dass die Feedbackwahrnehmungen der Schülerinnen und Schüler durch weitere personale, kontextuelle oder interaktionale Variablen (z. B. Lehrer-Schüler-Beziehung) verzerrt worden sind (vgl. Strijbos und Müller 2014). Die zuvor geschilderten Sachverhalte sollten daher bei der Interpretation der Befunde der vorliegenden Untersuchung und bei (möglichen) Rückschlüssen auf ein geschlechtsstereotypes Verhalten von Lehrkräften im Unterricht berücksichtigt werden. Ebenso spielen natürlich auch individuelle Merkmale der Schülerinnen und Schüler (z.B. Aussehen, Verhalten) eine nicht unerhebliche Rolle hinsichtlich der Wahrnehmungen und Erwartungen der Lehrkräfte und ihrem hiermit verbundenen Verhalten im Unterricht, die in der vorliegenden Untersuchung nicht mit in den Blick genommen wurden.

In weiterführenden Untersuchungen sollten Möglichkeiten der direkten Messung geschlechtsspezifischer Vorannahmen von Lehrkräften im Zusammenhang mit ihren Erwartungen und der hieraus resultierenden Feedbackerteilung im Unterricht geprüft werden. Denkbar wäre es, Lehrkräfte im Rahmen einer Fragebogenskala explizit zu ihren geschlechtsspezifischen Annahmen sowie den hiermit zusammenhängenden 
Erwartungen an Schülerinnen und Schüler zu befragen. Darüber hinaus könnte der Unterricht videographiert werden. Dieses Vorgehen würde eine deutlich differenziertere Erfassung der Interaktionen zwischen den Lernenden und Lehrenden im Unterricht ermöglichen. So könnte einerseits das von Lehrkräften an Schülerinnen und Schüler erteilte Feedback genauer erfasst werden. Andererseits könnten zudem auch nonverbale Rückmeldungen der Lehrerinnen und Lehrer in weiteren Untersuchungen berücksichtigt werden.

Funding Open Access funding enabled and organized by Projekt DEAL.

Open Access Dieser Artikel wird unter der Creative Commons Namensnennung 4.0 International Lizenz veröffentlicht, welche die Nutzung, Vervielfältigung, Bearbeitung, Verbreitung und Wiedergabe in jeglichem Medium und Format erlaubt, sofern Sie den/die ursprünglichen Autor(en) und die Quelle ordnungsgemäß nennen, einen Link zur Creative Commons Lizenz beifügen und angeben, ob Änderungen vorgenommen wurden.

Die in diesem Artikel enthaltenen Bilder und sonstiges Drittmaterial unterliegen ebenfalls der genannten Creative Commons Lizenz, sofern sich aus der Abbildungslegende nichts anderes ergibt. Sofern das betreffende Material nicht unter der genannten Creative Commons Lizenz steht und die betreffende Handlung nicht nach gesetzlichen Vorschriften erlaubt ist, ist für die oben aufgeführten Weiterverwendungen des Materials die Einwilligung des jeweiligen Rechteinhabers einzuholen.

Weitere Details zur Lizenz entnehmen Sie bitte der Lizenzinformation auf http://creativecommons.org/ licenses/by/4.0/deed.de.

\section{Literatur}

Boerma, I.E., Mol, S. E., \& Jolles, J. (2016). Teacher perceptions affect boys' and girls' reading motivation differently. Reading Psychology, 37(4), 547-569. https://doi.org/10.1080/02702711.2015.1072608.

Bos, W., Bremerich-Vos, A., Tarelli, I., \& Valtin, R. (2012). Lesekompetenzen im internationalen Vergleich. In W. Bos, I. Tarelli, A. Bremerich-Vos \& K. Schwippert (Hrsg.), IGLU 2011. Lesekompetenzen von Grundschulkindern in Deutschland im internationalen Vergleich (S. 91-136). Münster: Waxmann.

Burnett, P.C. (2002). Teacher praise and feedback and students' perceptions of the classroom environment. Educational Psychology, 22(1), 5-16. https://doi.org/10.1080/01443410120101215.

Cejka, M.A., \& Eagly, A.H. (1999). Gender-stereotypic images of occupations correspond to the sex segregation of employment. Personality and Social Psychology Bulletin, 25(4), 413-423. https://doi. org/10.1177/0146167299025004002.

Chen, Y., Thompson, M. S., Kromrey, J. D., \& Chang, G.H. (2011). Relations of student perceptions of teacher oral feedback with teacher expectancies and student self-concept. The Journal of Experimental Education, 79(4), 452-477. https://doi.org/10.1080/00220973.2010.547888.

Cohen, J. (1988). Statistical power analysis for the behavioral sciences (2. Aufl.). New York: Lawrence Erlbaum.

Cohen, J. (1992). A power primer. Psychological Bulletin, 112, 155-159. https://doi.org/10.1037/00332909.112.1.155.

Denn, A.-K., Lotz, M., Theurer, C., \& Lipowsky, F. (2015). „Prima, Lisa. Richtig“ und „Psst, Max. Hör auf zu stören!“ Eine quantitative Studie zu Unterschieden im Feedbackverhalten von Lehrkräften gegenüber Mädchen und Jungen im Mathematikunterricht des zweiten Schuljahres. Gender, 7(1), 29-47. https://doi.org/10.3224/gender.v7i1.18155.

Eccles, J.S. (2005). Subjective task value and the Eccles et al. model of achievement-related choices. In A. J. Elliot \& C. S. Dweck (Hrsg.), Handbook of competence and motivation (S. 105-121). New York: Guilford.

Ehm, J.-H., Duzy, D., \& Hasselhorn, M. (2011). Das akademische Selbstkonzept bei Schulanfängern. Spielen Geschlecht und Migrationshintergrund eine Rolle? Frühe Bildung, 0, 37-45. https://doi.org/ 10.1026/2191-9186/a000008. 
Faulstich-Wieland, H. (1995). Geschlecht und Erziehung. Grundlagen des pädagogischen Umgangs mit Mädchen und Jungen. Darmstadt: Wissenschaftliche Buchgesellschaft.

Gentrup, S., Rjosk, C., Stanat, P., \& Lorenz, G. (2018). Einschätzungen der schulischen Motivation und des Arbeitsverhaltens durch Grundschullehrkräfte und deren Bedeutung für Verzerrungen in Leistungserwartungen. Zeitschrift für Erziehungswissenschaft, 21(4), 867-891. https://doi.org/10.1007/s11618018-0806-2.

Gentrup, S., Lorenz, G., Kristen, C., \& Kogan, I. (2020). Self-fulfilling prophecies in the classroom: teacher expectations, teacher feedback and student achievement. Learning and Instruction. https://doi.org/10. 1016/j.learninstruc.2019.101296.

Glen, T., Heath, N.L., Karagiannakis, A., \& Hoida, D. (2004). Feedback practices in a sample of children with emotional and/or behavioral difficulties. Emotional and Behavioral Difficulties, 9(1), 54-69. https://doi.org/10.1177/1363275204041963.

Good, T.L., \& Brophy, J. (1978). Looking in Classrooms (2. Aufl.). New York: Harper and Row.

Good, T. L., Sterzinger, N., \& Lavigne, A. (2018). Expectation effects: Pygmalion and the initial 20 years of research. Educational Research and Evaluation, 24(3-5), 99-123. https://doi.org/10.1080/13803611. 2018.1548817.

Hannover, B. (2008). Vom biologischen zum psychologischen Geschlecht: Die Entwicklung von Geschlechterunterschieden. In A. Renkl (Hrsg.), Lehrbuch Pädagogische Psychologie (S. 339-388). Bern: Huber.

Hannover, B., \& Wolter, I. (2019). Geschlechtsstereotype: wie sie entstehen und sich auswirken. In B. Kortendiek, B. Riegraf \& K. Sabisch (Hrsg.), Handbuch Interdisziplinäre Geschlechterforschung (S. 201-210). Wiesbaden: Springer.

Hannover, B., Zander, L., \& Wolter, I. (2014). Entwicklung, Sozialisation und Lernen. In T. Seidel \& A. Krapp (Hrsg.), Pädagogische Psychologie (Bd. 6, S. 139-165). Weinheim, Basel: Beltz.

Hattie, J. (2009). Visible learning. A synthesis of over 800 meta-analyses relating to achievement. London, New York: Routledge.

Hattie, J., \& Timperley, H. (2007). The power of feedback. Review of Educational Research, 77(1), 81-112. https://doi.org/10.3102/003465430298487.

Hellmich, F. (2008). Erklärungsfaktoren für Geschlechterunterschiede in der Lesekompetenz bei Grundschulkindern am Ende ihrer Grundschulzeit. Zeitschrift für Grundschulforschung, 1(2), 46-58.

Helwig, R., Anderson, L., \& Indal, G. (2001). Influcence of elementary student gender on teachers' perceptions of mathematics achievement. The Journal of Educational Research, 95(2), 93-102. https:// doi.org/10.1080/00220670109596577.

Hornberg, S., Valtin, R., Potthoff, B., Schwippert, K., \& Schulz-Zander, R. (2007). Lesekompetenzen von Mädchen und Jungen im internationalen Vergleich. In W. Bos, S. Hornberg, K.-H. Arnold, G. Faust, L. Fried, E.-M. Lankes, K. Schwippert \& R. Valtin (Hrsg.), IGLU 2006. Lesekompetenzen von Grundschulkindern in Deutschland im internationalen Vergleich (S. 195-223). Münster: Waxmann.

Irvine, J. J. (1986). Teacher-student interactions: effects of student race, sex, and grade level. Journal of Educational Psychology, 78(1), 14-21. https://doi.org/10.1037/0022-0663.78.1.14.

Keller, C. (2001). Effect of teachers' stereotyping on students' stereotyping of mathematics as a male domain. The Journal of Social Psychology, 141(2), 165-173. https://doi.org/10.1080/00224540109600544.

Kessels, U., \& Heyder, A. (2017). Die Wertschätzung schulischer Anstrengung als Mediator von Geschlechtsunterschieden in Noten. Eine fächerspezifische Analyse. Zeitschrift für Entwicklungspsychologie und Pädagogische Psychologie, 49(2), 86-97. https://doi.org/10.1026/0049-8637/a000171.

Lenhard, W., \& Schneider, W. (2006). ELFE 1-6. Ein Leseverständnistest für Erst- bis Sechstklässler. Göttingen: Hogrefe.

Leonhart, R. (2009). Lehrbuch Statistik. Einstieg und Vertiefung (2. Aufl.). Bern: Huber.

Lindberg, S., Linkersdörfer, J., Ehm, J.-H., Hasselhorn, M., \& Lonnemann, J. (2013). Gender differences in children's math self-concept in the first years of elementary school. Journal of Education and Learning, 2(3), 1-8. https://doi.org/10.5539/jel.v2n3p1.

Ludwig, P.H. (2018). Erwartungseffekt. In D. H. Rost, J. R. Sparfeldt \& S. R. Buch (Hrsg.), Handwörterbuch Pädagogische Psychologie (5. Aufl. S. 141-146). Weinheim, Basel: Beltz.

McElvany, N. (2008). Förderung von Lesekompetenz im Kontext der Familie. Münster: Waxmann.

McElvany, N., Kessels, U., Schwabe, F., \& Kasper, D. (2017). Geschlecht und Lesekompetenz. In A. Hußmann, H. Wendt, W. Bos, A. Bremerich-Vos, D. Kasper, E.-M. Lankes, N. McElvany, T. C. Stubbe \& R. Valtin (Hrsg.), IGLU 2016. Lesekompetenzen von Grundschulkindern in Deutschland im internationalen Vergleich (S. 177-194). Münster: Waxmann.

McGeown, S. (2015). Sex or gender identity? Understanding children's reading choices and motivation. Journal of Research in Reading, 38(1), 35-46. https://doi.org/10.1111/j.1467-9817.2012.01546.x. 
McGeown, S., Goodwin, H., Henderson, N., \& Wright, P. (2011). Gender differences in reading motivation: does sex or gender identity provide a better account? Journal of Research in Reading, 35(3), 328-336. https://doi.org/10.1111/j.1467-9817.2010.01481.x.

Neitzel, C., \& Davis, D. (2014). Direct and indirect effects of teacher instruction and feedback on student adaptive help-seeking in upper-elementary literacy classrooms. Journal of Research in Education, 24(1), 53-68.

Niklas, F., \& Schneider, W. (2012). Die Anfänge geschlechtsspezifischer Leistungsunterschiede in mathematischen und schriftsprachlichen Kompetenzen. Zeitschrift für Entwicklungspsychologie und Pädagogische Psychologie, 44(3), 123-138. https://doi.org/10.1026/0049-8637/a000064.

Pintrich, P.R., \& Schunk, D.H. (2002). Motivation in education. Theory, research, and applications (2. Aufl.). New Jersey: Merrill Prentice Hall.

Retelsdorf, J., Schwartz, K., \& Asbrock, F. (2015). "Michael can’t read!" Teachers' gender stereotypes and boys' reading self-concept. Journal of Educational Psychology, 107(1), 186-194. https://doi.org/10. 1037/a0037107.

Robinson-Cimpian, J.P., Lubienski, S.T., Ganley, C.M., \& Copur-Gencturk, Y. (2014). Teachers' perceptions of students' mathematics proficiency may exacerbate early gender gaps in achievement. Developmental Psychology, 50(4), 1262-1281. https://doi.org/10.1037/a0035073.

Rosenthal, R., \& Jacobsen, L. (1971). Pygmalion im Unterricht: Lehrererwartungen und Intelligenzentwicklungen der Schüler. Weinheim: Beltz.

Schabmann, A., Landerl, K., Bruneforth, M., \& Schmidt, B. M. (2012). Lesekompetenz, Leseunterricht und Leseförderung im österreichischen Schulsystem. Analysen zur pädagogischen Förderung der Lesekompetenz. In B. Herzog-Punzenberger (Hrsg.), Fokussierte Analysen bildungspolitischer Schwerpunktthemen. Nationaler Bildungsbericht Österreich 2012, (Bd. 2, S. 17-69). Graz: Leykam.

Schöne, C., Dickhäuser, O., Spinath, B., \& Stiensmeier-Pelster, J. (2002). Skalen zur Erfassung des schulischen Selbstkonzepts. SESSKO. Göttingen: Hogrefe.

Simpson, A.W., \& Erickson, M. T. (1983). Teachers' verbal and nonverbal communication patterns as a function of teacher race, student gender, and student race. American Educational Research Journal, 20(2), 183-198. https://doi.org/10.3102/00028312020002183.

Stahns, R., \& Rieser, S. (2018). Qualität des Leseunterrichts in vierten Klassen in der Grundschule unter den Bedingungen von Mehrsprachigkeit. Ergebnisse der Lehrkräftebefragung von IGLU 2011. Zeitschrift für Grundschulforschung, 11(1), 131-145. https://doi.org/10.1007/s42278-018-0007-3.

Strijbos, J.-W., \& Müller, A. (2014). Personale Faktoren im Feedbackprozess. In H. Ditton \& A. Müller (Hrsg.), Feedback und Rückmeldungen. Theoretische Grundlagen, empirische Befunde, praktische Anwendungsfelder (S. 83-134). Münster: Waxmann.

Stutz, F., Schaffner, E., \& Schiefele, U. (2017). Measurement invariance and validity of a brief questionnaire on reading motivation in elementary students. Journal of Research in Reading, 40(4), 439-461. https://doi.org/10.1111/1467-9817.12085.

Tiedemann, J. (2000). Parents' gender stereotypes and teachers' beliefs as predictors of children's concept of their mathematical ability in elementary school. Journal of Educational Psychology, 92(1), 144-151. https://doi.org/10.1037/0022-0663.92.1.144.

Weinstein, R.S., Marshall, H.H., Brattesani, K.A., \& Middlestadt, S.E. (1982). Student perceptions of differential teacher treatment in open and traditional classrooms. Journal of Educational Psychology, 74, 678-692. https://doi.org/10.1037/0022-0663.74.5.678.

Wendt, H., Steinmayr, R., \& Kasper, D. (2016). Geschlechterunterschiede in mathematischen und naturwissenschaftlichen Kompetenzen. In H. Wendt, W. Bos, C. Selter, O. Köller, K. Schwippert \& D. Kasper (Hrsg.), TIMSS 2015. Mathematische und naturwissenschaftliche Kompetenzen von Grundschulkindern in Deutschland im internationalen Vergleich (S. 257-297). Münster: Waxmann. 